\title{
An Investigation of Coupled and Decoupled Iterative Algorithms for Energy Balance Calculations
}

\author{
Y. Apanovich, E. Lyumkis, B. Polsky, and P. Blakey
}

Algorithms Development Group, Silvaco International 4701 Patrick Henry Drive, Bldg. 3, Santa Clara, CA 95054, USA

\begin{abstract}
Coupled and decoupled iterative algorithms for the solution of nonlocal transport models are investigated. The decoupled scheme described in [1] exhibits excellent convergence properties except under strong breakdown conditions, where the full Newton method is required. Simulation results of snap-back curves for submicron BJT and MOS devices illustrate the potential of both algorithms.
\end{abstract}

\section{Introduction}

Deterministic nonlocal transport models are widely used for the analysis of transport phenomena in submicron devices. These models provide a description of effects that are neglected by the conventional drift-diffusion model (DDM). The effects include velocity overshoot and nonlocal impact ionization. These nonlocal models are commonly referred to as energy balance (EB), energy transport (ET) or hydrodynamic (HD) models. This class of models will be referred to here as 'energy balance' models.

From the numerical point of view device simulation using energy balance models is more complicated than simulation based on the DDM. Additional partial differential equations ( $P^{2} E^{\prime} s$ ) must be solved, and the system of PDE's exhibits greater nonlinearity. A natural decoupled iterative algorithm for energy balance models is as follows: the electron and hole continuity equations and Poisson's equation are solved by either Gummel or Newton methods with the carrier temperatures held constant; and then electron and hole temperatures are updated from the solution of the energy balance equations. This approach is added easily to existing general purpose device simulators, and is therefore widely used.

Meinerzhagen et. al. [1] have shown that the convergence rate of this algorithm is slow for high biases, and that for some cases convergence may 
not be obtained at all. They suggested a new algorithm that differs from the previous one at the second step. In the new algorithm the energy balance equation is solved simultaneously with the carrier continuity equation. A dramatic increase in convergence rate for MOS transistor calculations is obtained. At the moment this algorithm seems to be the best available for unipolar simulations. The work described here was performed to investigate the convergence rate of Meinerzhagen's method for bipolar simulations, especially in the strong breakdown region; and to establish whether it is necessary to also provide the full Newton algorithm in a truly general purpose device simulator.

\section{Energy Balance Model}

The variant of the energy balance model used in the present work follows [2], and was described in [3]. The impact ionization coefficient for electron was modeled as:

$$
\alpha_{n}=a_{n} \exp \left(-\frac{b_{n}}{E_{\text {eff }}}\right),
$$

with an and $b_{n}$ taken from [4], and an effective electric field determined from the relationship:

$$
\mathrm{E}_{\text {eff }}=\frac{3}{2} \frac{\mathrm{kT}}{\mathrm{q} \lambda_{\mathrm{n}}},
$$

where $\lambda_{n}$ is the energy relaxation length for electrons. A similar expression is used for $\alpha_{p}$.

\section{Methods}

Meinerzhagen's method and the full Newton method for all five equations were implemented in SPISCES. Meinerzhagen's algorithm is implemented in the following form. For each outer loop, the electron and hole continuity equations and Poisson's equation are solved for either a specified number of iterations, or to convergence, using Newton iteration. One Newton iteration is then performed for the simultaneous solution of the electron continuity and electron temperature equations, after which one Newton iteration is performed for the simultaneous solution of the hole continuity and hole temperature equations. (This implementation differs slightly from [1] where only one Newton iteration was performed at the first step. This does not impact the asymptotic behavior of the convergence rate.) All linearized systems of equations are solved with a direct solver.

\section{Results}

Numerical experiments were performed to establish the behavior of the different algorithms for several situations. The first experiment was for a submicron bipolar transistor similar to the device considered in [5]. The 
emitter-base voltage was kept constant and equal to $-0.75 \mathrm{~V}$. An external resistance of $1.0 \mathrm{e} 5 \Omega \cdot \mu \mathrm{m}$ was connected between the collector and a voltage source $\mathrm{V}_{\mathrm{cc}}$. Figure 1 shows the calculated collector current as a function of collector-base voltage. Figure 2 shows the error versus iteration number for different values of $V_{c c}$ : curve 1 is for $V_{c c}=8.5 \mathrm{~V}$, curve 2 for $V_{c c}=10.5 \mathrm{~V}$, and curve 3 for $V_{C C}=11 \mathrm{~V}$, all calculated using Meinerzhagen's method with the equations solved to convergence in the first step. The corresponding collector-base voltages were 7.99, 9.54 and $9.87 \mathrm{~V}$ respectively. Curve 4 is for $\mathrm{V}_{\mathrm{cc}}=11 \mathrm{~V}$, calculated using the full Newton method. Errors are measured from the fully converged solution obtained using Newton method with very tight convergence criteria. This figure shows that the rate of convergence of Meinerzhagen's algorithm is excellent for $\mathrm{V}_{\mathrm{cc}}=8.5 \mathrm{~V}$, but that it decreases rapidly near the snap-back voltage. The Newton algorithm exhibits similar behavior to that shown in Figure 2 for all biases.

A second experiment was for a conventional MOS device with a channel length of $0.45 \mu \mathrm{m}$. The gate, source and substrate voltages were grounded. An external resistance of $1 . e 10 \Omega \cdot \mu \mathrm{m}$ was connected between the drain, and a voltage source $V_{d d}$. The drain current as a function of drain voltage is shown in Figure 3. Errors versus iteration number for different values of $V_{d d}$ are shown in Figure 4 . Curve 1 is for $V_{d d}=12.25 \mathrm{~V}$, curve 2 is for $\mathrm{V}_{\mathrm{dd}}=12.4125 \mathrm{~V}$, curve 3 is for $\mathrm{V}_{\mathrm{dd}}=12.425 \mathrm{~V}$, and curve 4 is for $\mathrm{V}_{\mathrm{dd}}=12.425 \mathrm{~V}$. The corresponding drain voltages were 12.246, 12.364 and $12.362 \mathrm{~V}$ respectively. As before, the first three curves are calculated using Meinerzhagen's method with the equations solved to convergence in the first step, and curve 4 is obtained using full Newton iteration. The decoupled algorithm shows fast convergence for $\mathrm{V}_{\mathrm{dd}}=12.25 \mathrm{~V}$, but fails near the snap-back voltage.

\section{Conclusions}

Meinerzhagen's decoupled algorithm [1] exhibits excellent convergence properties for pre-breakdown two carrier, two temperature simulations. The convergence rate decreases rapidly under strong breakdown conditions. The full Newton method is required to handle such conditions. Meinerzhagen's algorithm and the full Newton method will both be available in future releases of the SPISCES and BLAZE device simulators.

\section{References}

[1] B. Meinerzhagen, K. H. Bach, I. Bork and W. L. Engl, "A New Highly Efficient Nonlinear Relaxation Scheme for Hydrodynamic MOS Simulations," NUPAD IV Abstracts, Seattle, USA, pp. 91-96, 1992.

[2] R. Stratton, "Diffusion of Hot and Cold Electrons in Semiconductor Barriers," Phys. Rev., vol. 126, pp. 2002-2013, 1962. 
[3] Y. Apanovich, E. Lyumkis, B. Polsky, A. Shur, and P. Blakey, "Numerical Simulation of Submicron Devices Using Energy Balance and Hydrodynamic Models in the General Purpose Device Simulator SPISCES-2B," Abstracts of the International Workshop on Computational Electronics, University of Illinois, May 28-29, pp. 95-98, 1992.

[4] R. Van Overstraeten and H. DeMan, "Measurement of the Ionization Rates in Diffused Silicon p-n Junctions," Solid-State Electronics, vol. 13, pp. 583-608, 1970.

[5] E. F. Crabble, J. M. S. Stork, G. Baccarani, M. V. Fischetti, and S. E. Laux, "The Impact of Non-equilibrium Transport on Breakdown and Transit Time in Bipolar Transistors," IEDM Tech. Dig., pp. 463-466, 1990.

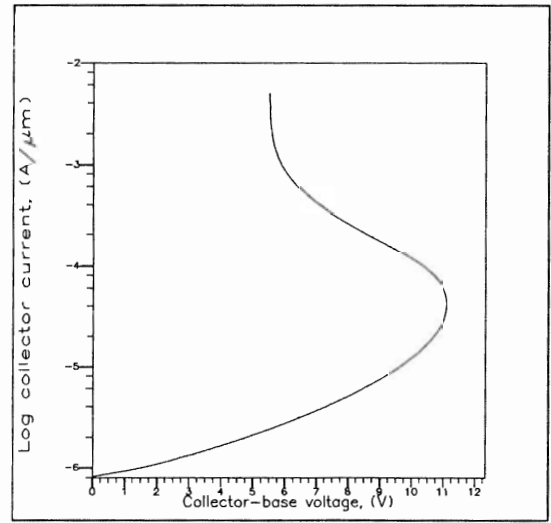

Figure 1.

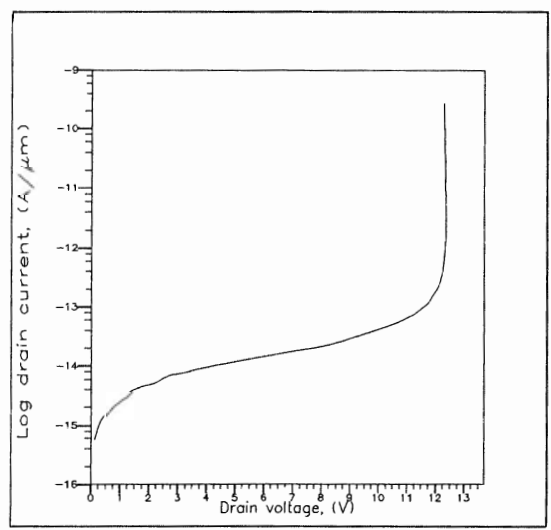

Figure 3.

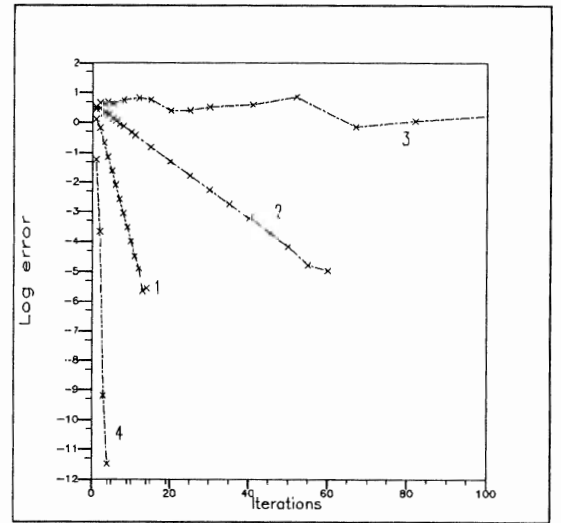

Figure 2.

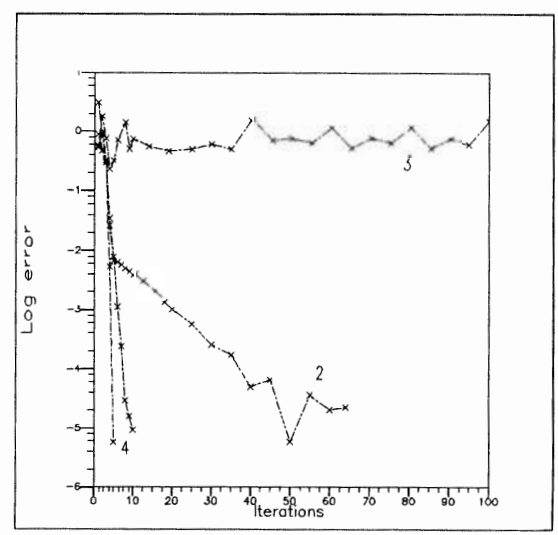

Figure 4. 